\title{
DEBATES
}

\section{Una Aproximación al Estudio de la Actividad Distrital de los Diputados: los Casos de Chile y Bolivia}

\author{
An Aproach to the Study of Congres Representatives Distrital \\ Activity: the Cases of Chile and Bolivia
}

\section{Mikel Barreda \\ Leticia M. Ruiz}

\section{Resumen}

El paper se ocupa de la actividad de los diputados en sus distritos/circunscripciones en períodos no electorales. Se presta especial atención a la autonomía del diputado con respecto al partido en la definición de las actividades de representación en el distrito por el que fue electo. Dos son los objetivos principales del trabajo. En primer lugar, realizar una descripción de la actividad distrital, a partir de un análisis de la frecuencia, soportes y relación con el partido durante la actividad distrital. En segundo lugar, explorar algunos de los posibles determinantes del grado de autonomía de los diputados con respecto a sus partidos en su actividad distrital. Los casos elegidos son partidos de Bolivia y Chile.

\section{Palabras clave}

Representación Política; Actividad Distrital; Diputados; Bolivia; Chile.

\begin{abstract}
The paper studies congressmen work in their districts/constituencies once elections have taken place. Special attention is given to the degree of congressmen autonomy with regard to their parties. Two are the goals of this paper. The first goal is to offer a description of the types o activities of representation that congressmen carry out in their districts. The second goal is to explore the determinants of congressmen degree of autonomy. The cases chosen are political parties from Bolivia and Chile.
\end{abstract}

\section{Keywords}

Political Representation; District activity; Congressmen; Bolivia; Chile. 


\section{Introducción ${ }^{1}$}

Este trabajo se ocupa de una de las posibles avenidas de estudio de la tarea de representación. Se centra en el modo en que los diputados mantienen sus vínculos con el electorado de sus circunscripciones en períodos no electorales. Los estudios sobre clientelismo son prácticamente los únicos que han examinado la actividad de los diputados en sus distritos y, además, han tendido a centrarse en el momento electoral. De ahí que sea insuficiente la evidencia empírica disponible para formular una teoría general de la movilización partidista que permita comprender el modo en que los representantes se conectan con sus representados (HAGOPIAN, 2007).

En este contexto, nuestro trabajo aporta nuevos datos para la reflexión sobre la actividad distrital y sus determinantes. La actividad distrital se refiere al conjunto de acciones que llevan a cabos los diputados en sus circunscripciones para mantener los vínculos con sus electores. Esta actividad puede tener, además, una finalidad a medio plazo que es prepararse - silas circunstancias institucionales, personales y partidistas lo permiten - para una posible reelección.

Dos son los objetivos de este estudio. El primero es realizar una descripción de la actividad distrital, que analice la frecuencia, soportes y relación con el partido durante la actividad distrital. El segundo objetivo es explorar algunos de los posibles determinantes del grado de autonomía de los diputados con respecto a sus partidos en su actividad distrital.

La mayor parte de los datos son primarios y proceden de encuestas a asesores parlamentarios de cinco partidos políticos de Chile (legislatura 2009-2013) y Bolivia (legislatura 2010-2015). Estas encuestas (un total de cuarenta y seis entrevistas en Bolivia, y veinticinco en Chile) se realizaron durante los meses de febrero, marzo y abril de 2013.

\section{El estudio de la actividad distrital}

La actividad distrital constituye un ámbito nuevo de estudio de la política latinoamericana. Los diputados llevan a cabo un conjunto diverso de acciones en los lugares por los que fueron elegidos con la finalidad de mantener su presencia y contactos con los electores y trabajar, eventualmente, para su reelección. Se trata de una actividad sostenida y habitual entre todos los diputados, si bien varía en estilos e intensidad. Con el estudio de la actividad distrital se arroja luz al modo en que se lleva a cabo una de las aristas de la tarea de representación: conocer al electorado e

\footnotetext{
${ }^{1}$ Este trabajado ha sido financiado por el proyecto "Los vínculos entre electores y partidos: la actividad distrital de los diputados de Chile, Perú y Bolivia” (CICYT, CSO2011-24344). Una versión preliminar fue presentada en el XXXI Congreso de LASA, Washington, 29 mayo a 1 junio de 2013.
} 
interaccionar con él. Junto al ámbito distrital, otros escenarios donde el parlamentario lleva a cabo la tarea de representación son el parlamento y su propio partido político. En ellos también se pueden estudiar los vínculos entre representantes y representados.

Este tipo de aproximaciones al estudio de la representación ha sido frecuente en Estados Unidos. Uno de los trabajos pioneros es el de Fenno (2003), en el que se examina lo que hacen los representantes en sus circunscripciones para mantener los vínculos con sus electores. Por el contrario, en América Latina este tipo de aproximaciones es muy limitado. Hasta la fecha, los trabajos sobre clientelismo en América Latina son de los pocos que han considerado las actividades que llevan a cabo los diputados y los partidos en sus circunscripciones. Los trabajos de Helmke y Levitsky (2006), Brusco, Nazareno y Stokes (2004) y el de Amaral y Stokes (2005), por citar algunos de ellos, detallan las estrategias de los partidos, a la vez que anticipan el modo en que éstas afectan a la tarea futura de representación, así como sus efectos sobre las dinámicas partidistas y sobre la accountability de los sistemas políticos. Sin embargo, estos estudios se centran en períodos electorales, que están marcados por el objetivo de maximizar el rendimiento electoral. De manera que se sabe poco de la actividad de los diputados en períodos ordinarios.

Aunque no se dispone de investigaciones específicas sobre actividad distrital en América Latina, diversos estudios sobre los representantes políticos de la región han generado algunas expectativas teóricas y empíricas sobre la actividad distrital, tales como el control que mantienen algunos partidos sobre sus diputados o la combinación de estrategias que desarrollan los diputados para vincularse con sus electores. Cabe destacar, entre otros, los trabajos de Alcántara (2007) sobre preferencias de los diputados; de Carey (2009) y Morgenstern y Nacif (2002) sobre su comportamiento en el legislativo; de Kitschelt et al. (2010) sobre vínculos entre representantes y representados - quepropone la existencia de vínculos personalistas, programático-partidistas y clientelares - asícomo el de Roberts (2002) sobre esta misma temática; y de Siavelis y Morgenstern (2008) sobre carreras políticas de los diputados. Estos últimos proponen una tipología de candidatos a diputados ("servidores al distrito", "leales al partido", "empresarios políticos" y "delegados de grupo”) que será utilizada en este estudio sobre diputados.

En estas circunstancias, el presente trabajo sobre la actividad distrital de los diputados en periodos no electorales abre una veta de investigación sobre la representación política en América Latina. En concreto, el estudio analiza la actividad distrital de los diputados de tres partidos políticos chilenos ( $\mathrm{RN}$ - Renovación Nacional -, PPD - Partido por la Democracia - y PDC - Partido Demócrata Criastiano) y dos bolivianos (MAS - Movimiento al Socialismo - y PPB-CN - Plan 
Progreso para Bolivia - ConvergenciaNacional) que son los partidos o coaliciones con más diputados en el período legislativo objeto de estudio. Para ello, se realizó una encuesta (en 2013) a asesores parlamentarios (ver Anexo 1 para distribución del $n$ de las encuestas y otros detalles del cuestionario y de la ficha técnica). Los tres partidos chilenos incluidos tienen una considerable estructuración programática y una presencia continuada en la Cámara de Diputados, donde constituyen grupos parlamentarios numerosos. El más antiguo de los tres partidos chilenos considerados es el PDC que, junto con el PPD, pertenecen a la misma la coalición de centroizquierda, que en el momento de realizar las encuestas se denominaba Concertación de Partidos por la Democracia. Ambos partidos convergen en su posición ante el denominado "clivaje Pinochet", así como en materia social y económica, aunque difieren en cuestiones de valores: el PDC mantiene posiciones más conservadoras y el PPD posiciones más progresistas. Por su parte, RN forma parte de la coalición de derecha Alianza por Chile que estaba en el gobierno de Piñera (2010-2014) cuando se realizaron las entrevistas. Se trata de un partido con un programa conservador en materia social, económica y de valores, y cuyos cuadros se han desmarcado en numerosas ocasiones del legado Pinochet. Respecto a los partidos bolivianos seleccionados predomina la estructuración personalista. El MAS constituye un ejemplo de movimiento local de oposición que se institucionaliza y logra articularse a nivel nacional y deja atrás su labor de oposición, al ocupar ininterrumpidamente el gobierno desde el 2005 bajo la presidencia de Morales y con una amplia mayoría en la Cámara de Diputados. Sus reivindicaciones sociales y su transformación institucional del país lo asimilan a otros ejemplos de izquierda populista en la región. Por su parte, el PPB ha funcionado como el principal grupo opositor al MAS. Con una ideología de derecha, esta coalición logró conquistar el voto de los electores contrarios al proceso de transformación llevado a cabo por Morales, aunque no competirá en las elecciones de 2014 de forma unida.

Por otra parte, los dos países a los que pertenecen los diputados encuestados constituyen casos muy dispares en cuanto a sus procesos de representación y sistemas de partidos. Así, Bolivia es uno de los países latinoamericanos que ha experimentado de forma más acusada las consecuencias de un modelo fallido de representación, con procesos de desafección creciente, volatilidad, fragmentación, y fluidez (MAINWARING, BEJARANO y PIZARRO, 2006). En la actualidad cuenta con un sistema de partidos menos volatil debido a la concentración de voto en torno al MAS, pero la inestabilidad del resto de oferta partidista junto los niveles de descrédito que sufren los partidos en Bolivia nos impiden considerarlo como un sistema de partidos institucionalizado, además de presentar niveles bajos de nacionalización. En el caso de 
Chile, aunque ha visto aumentar el número de abstencionistas y desafectos con el sistema y aún cuando atraviesa un proceso de cierta desestructuración programática, todavía constituye uno de los sistemas de partidos más estables de la región (LUNA y MARDONES, 2010) y de los que presenta mayores niveles de instituiconalización y menores niveles de nacionalización. Al igual que Bolivia, Chile tiene altos niveles de fragmentación y de polarización. Así mismo, los dos países difieren en su arquitectura institucional ${ }^{2}$. A efectos de nuestro objeto de estudio, destaca el distinto modo de elegir a sus diputados. Chile es un sistema de reparto mayoritario con circunscripciones binominales y una alta barrera legal para que un mismo partido consiga los dos diputados. Por su parte, en Bolivia se combina la elección de diputados mediante sistema mayoritario con otros diputados elegidos mediante representación proporcional. Además, los diputados chilenos tienen la posibilidad de reelección indefinida, mientras que los bolivianos cuentan con una limitación de dos mandatos. De ahí el interés de obtener una visión comparada de la actividad distrital en contextos tan diferentes que condicionan el grado de personalización del voto y la relación entre representantes y representados ${ }^{3}$.

Vistos estos contrastes entre los dos casos, la expectativa inicial del presente estudio es que, además de la diversidad de soportes e intensidad de la actividad distrital, existan diferencias respecto al grado de autonomía partidista de la actividad distrital; diferencias que se espera encontrar tanto al establecer la comparación entre partidos como al examinar el interior de los mismos. Es esperable que la actividad distrital no sea homogénea dentro de aquellos partidos en los que conviven diversos modos de mantener vínculos y llevar a cabo interacciones con su electorado.

\section{La actividad distrital en Bolivia y Chile}

\section{- Frecuencia y naturaleza de la actividad distrital}

La actividad distrital es un aspecto contemplado en los reglamentos de la Cámara Baja tanto de Chile como de Bolivia. En ellos se reserva la última semana de cada mes al contacto con los electores de la circunscripción. Esta semana distrital en la que se aparca el trabajo netamente legislativo asegura que todos los diputados viajen, al menos, una vez al mes a sus circunscripciones. De todos modos, la intensidad de las visitas a los distritos es superior al mínimo establecido en los reglamentos (ver Tabla 1), de manera que los diputados suelen tener una fuerte presencia en los distritos por los que fueron elegidos. La mayor parte de los diputados

\footnotetext{
${ }^{2}$ Sobre los contrastes entre sistemas de partidos en estas dimensiones, ver Ruiz (2013).

${ }^{3}$ Sobre estas cuestiones ver Negretto (2013).
} 
de los cinco partidos acuden a su distrito todas las semanas. Por otra parte, se aprecia un mayor número de visitas entre los diputados de partidos chilenos que bolivianos. De los partidos entrevistados, la tendencia encontrada apuntaría a que los diputados de RN y PDC son los tienen más presencia en los distritos. Esta conclusión sobre RN concuerda con la imagen tradicional de sus parlamentarios como personalidades independientes con fuerte liderazgo y actividad en sus circunscripciones.

Tabla 1 - Frecuencia de visitas del diputado a su circunscripción electoral y/o contacto con los ciudadanos (en \%)

\begin{tabular}{c|c|c|c|c|c}
\hline & MAS & PPB-CN & PDC & RN & PPD \\
\hline Todas las semanas & 67,9 & 73,3 & 88,9 & 100 & 80 \\
\hline Varias veces por semana & 14,3 & & 11,1 & & 20 \\
\hline Una vez cada quince días & 7,1 & 13,3 & & & \\
\hline Una vez al mes & 10,7 & 13,3 & & & \\
\hline $\begin{array}{c}\text { Promedio días del diputado } \\
\text { en circunscripción }\end{array}$ & 9 & 8 & 14,8 & 16,1 & 12,4 \\
\hline$(\mathrm{N})$ & 28 & 15 & 9 & 7 & 10 \\
\hline
\end{tabular}

Fuente: Elaboración propia con datos del proyecto CSO2011-24344.

Ahora bien, ¿qué suelen hacer los diputados en los distritos para mantener su presencia? Los datos de los que se dispone hasta el momento muestran que los diputados carecen de una estrategia selectiva o específica muy marcada. En general, los parlamentarios de los cinco partidos políticos llevan a cabo actividades de índole variada. La Tabla 2 presenta en gris aquellas actividades distritales que están relacionadas directamente con el partido (reuniones con líderes de partido a nivel local, reuniones con militantes del partido y reuniones con líderes del partido a nivel nacional), en contraste con el resto de actividades que el diputado puede que haya organizado de forma autónoma con respecto al partido (inauguración de obras o visitas oficiales, actos organizados individualmente en el distrito, entrevistas en medios). Por países, los datos muestran que los diputados bolivianos suelen tener más actividades partidistas que actividades autónomas con respecto del partido; mientras que los diputados chilenos presentan un reparto más equitativo de su actividad entre aquéllas relacionadas con el partido y aquéllas organizadas al margen de éste. Así, el MAS y el PPB tienen una mayor número de actividades partidistas. Por su parte, los diputados de PDC y RN se reparten prácticamente de forma similar entre un tipo y otro de actividades, con una ligera inclinación hacia las partidistas. Los actuales problemas organizativos del PDC hacen de la actividad distrital un tema especialmente sensible, ya que en el pasado tenía una presencia con sedes locales y cuadros propios en todo el país que ha perdido y necesita reconquistar (HUNNEUS, 
2003). En lo que se refiere a los diputados del PPD, también tiene una estrategia mixta pero en este caso con una ligera inclinación hacia las actividades autónomas respecto al partido. Esta conclusión sobre el PPD casa con la argumentación de Plumb (1998) sobre el devenir de este partido como reflejo de una nueva cultura organizativa más pragmática, donde el partido es un espacio que permite fortalecer los liderazgos individuales.

Tabla 2 - Frecuencia de distintas actividades de representación en los distritos electorales por parte de los diputados (promedios: 1 nunca -4 mucha frecuencia)

\begin{tabular}{c|c|c|c|c|c}
\hline & MAS & PPB-CN & PDC & RN & PPD \\
\hline $\begin{array}{c}\text { Inauguración de instalación pública o } \\
\text { visita oficial sólo o en compańía de } \\
\text { otras autoridades locales }\end{array}$ & $\begin{array}{c}2,8 \\
(25)^{*}\end{array}$ & $\begin{array}{c}2,7 \\
(13)\end{array}$ & $\begin{array}{c}3,4 \\
(9)\end{array}$ & $\begin{array}{c}4 \\
(7)\end{array}$ & 3,4 \\
& & & & & \\
\hline Ha organizado individualmente & 2,8 & 3 & 3,6 & 3,9 & 3,7 \\
algún acto con electores del distrito & $(25)$ & $(14)$ & $(9)$ & $(7)$ & $(10)$ \\
\hline Suele ser entrevistado en la prensa & 2,8 & 2,8 & 3,3 & 3,7 & 3,9 \\
local o radio de su distrito & $(25)$ & $(14)$ & $(9)$ & $(7)$ & $(10)$ \\
\hline Se ha reunido con líderes de su & 3,2 & 3,1 & 4 & 3,9 & 3,7 \\
partido a nivel local & $(27)$ & $(11)$ & $(9)$ & $(7)$ & $(10)$ \\
\hline Se ha reunido con líderes de su & 3,4 & 3,4 & 3,2 & 4 & 3 \\
partido a nivel nacional & $(26)$ & $(15)$ & $(9)$ & $(7)$ & $(10)$ \\
\hline Se ha reunido con militantes de su & 3,2 & 3,2 & 3,3 & 3,9 & 3,9 \\
partido a nivel local & $(25)$ & $(14)$ & $(9)$ & $(7)$ & $(10)$ \\
\hline
\end{tabular}

* El N se apresenta entre parenteses.

Fuente: Elaboración propia con datos del proyecto CSO2011-24344.

Si se analizan de forma desagregada las actividades relacionadas únicamente con el partido, se aprecia que los diputados chilenos participan con mayor frecuencia que los diputados bolivianos en actividades con los líderes locales del partido, así como con los militantes del partido. En cambio, los bolivianos tienen más actividades con los miembros nacionales del partido.

Una de las razones de la actividad de los diputados en la circunscripción sería el conocimiento de los problemas de la misma. Esta conclusión constituye una tendencia evidente en los datos que aquí se presentan. La Tabla 3 muestra que los contactos con autoridades de la circunscripción, con el electorado y con las asociaciones de la circunscripción son los primeros canales que suelen utilizar los diputados para conocer los problemas de la circunscripción. Ello sugeriría que, en este punto, todos los diputados funcionan como "servidores del distrito", utilizando la clasificación que elaboraron Siavelis y Morgenstern (2008) para analizar los perfiles de los candidatos a diputados. A su vez, destaca el papel sólo complementario de los 
medios de comunicación: en el caso boliviano no supera el 20\% en ninguno de los dos partidos como primera opción y en el caso chileno no recibe ninguna respuesta en primera opción. Hay otras dos ausencias notables en estas respuestas. Por una parte, el partido ocupa un papel prácticamente nulo para informarse de los problemas de la circunscripción. Sólo un 14,3\% de los diputados del MAS lo eligen como segunda fuente más utilizada. Por otra parte, se registra un uso residual de Internet y de las redes sociales con el objetivo de conocer los problemas de la circunscripción. Únicamente recurren a Internet los diputados del PPD y de PPB-CN y de una forma bastante limitada.

Tabla 3 - Fuentes de información más utilizadas ( $1^{\mathrm{a}}$ y $2^{\mathrm{a}}$ opción) para que el diputado conozca los problemas de su circunscripción (en \%)

\begin{tabular}{|c|c|c|c|c|c|c|}
\hline & & MAS & $\begin{array}{c}\text { PPB- } \\
\text { CN }\end{array}$ & PDC & $\mathrm{RN}$ & PPD \\
\hline \multirow{2}{*}{ Medios de comunicación tradicionales } & $1^{a}$ & 7,1 & 20 & & & \\
\hline & $2^{a}$ & 7,1 & 33,3 & 11,1 & & 50 \\
\hline \multirow{2}{*}{$\begin{array}{l}\text { Conversaciones con otros diputados de } \\
\text { circunscripción }\end{array}$} & $1^{a}$ & & 13,3 & & & \\
\hline & $2^{a}$ & & & 11,1 & & \\
\hline \multirow{2}{*}{$\begin{array}{l}\text { Contactos con autoridades locales o } \\
\text { provinciales de circunscripción }\end{array}$} & $1^{\mathrm{a}}$ & 60,7 & 33,3 & 11,1 & 42,9 & \\
\hline & $2^{a}$ & 28,6 & 26,7 & & 42,9 & \\
\hline \multirow{2}{*}{$\begin{array}{l}\text { Contactos con electores de } \\
\text { circunscripción }\end{array}$} & $1^{a}$ & 7,1 & 6,7 & 66,7 & 14,3 & 90 \\
\hline & $2^{a}$ & 10,7 & 20 & 22,2 & 14,3 & 20 \\
\hline \multirow{2}{*}{$\begin{array}{c}\text { Contactos con asociaciones ciudadanas } \\
\text { y lo grupos de interés de la } \\
\text { circunscripción }\end{array}$} & $1^{\mathrm{a}}$ & 25,0 & 20 & 22,2 & 42,9 & \\
\hline & $2^{a}$ & 39,3 & 20 & 55,6 & 42,9 & 10 \\
\hline \multirow{2}{*}{ Internet y redes sociales } & $1^{a}$ & & 6,7 & & & 10 \\
\hline & $2^{a}$ & & & & & 20 \\
\hline \multirow{2}{*}{$\begin{array}{c}\text { Reuniones con miembros del partido a } \\
\text { nivel local o provincial }\end{array}$} & $1^{a}$ & & & & & \\
\hline & $2^{a}$ & 14,3 & & & & \\
\hline \multicolumn{2}{|l|}{$(\mathrm{N})$} & 28 & 15 & 9 & 7 & 10 \\
\hline
\end{tabular}

Fuente: Elaboración propia con datos del proyecto CSO2011-24344.

Junto a las visitas a la circunscripción por parte de los diputados, las visitas de los electores a las oficinas del Congreso es otro modo de mantener contacto con la circunscripción que se puede englobar dentro de la actividad distrital. Nuevamente aquí se aprecia una tendencia de contraste entre los diputados de los partidos chilenos y de los partidos bolivianos (Tabla 4). Éstos últimos registran un mayor número de visitas de electores en sus oficinas, algunos incluso más de veinte visitas a la semana. Por el contrario, los diputados chilenos tienen una menor actividad de atención al electorado en la sede del Congreso. 
Tabla 4 - Frecuencia de visitas a la oficina del diputado en el Congreso por parte de electores de su circunscripción (en\%)

\begin{tabular}{c|c|c|c|c|c}
\hline & MAS & PPB-CN & PDC & RN & PPD \\
\hline Ninguna & & 6,7 & & & \\
\hline Menos de 5 a la semana & 28,6 & 20 & 66,5 & 57,1 & 60 \\
\hline Entre 5 y 10 a la semana & 42,9 & 33,3 & 33,3 & 14,3 & 40 \\
\hline Entre 10 y 20 & 10,7 & 26,7 & 33,3 & & \\
\hline Más de 20 & 17,9 & 6,7 & & & \\
\hline No lo sé & & 6,7 & & 28,6 & \\
\hline (N) & 28 & 15 & 9 & 7 & 10 \\
\hline
\end{tabular}

Fuente: Elaboración propia con datos del proyecto CSO2011-24344.

Así mismo, la Tabla 5 muestra que el objetivo más habitual de las visitas de los electores al Congreso es informar de problemas en la circunscripción. Esta práctica es muy marcada entre el electorado del MAS en Bolivia y del PDC y PPD en Chile. Junto a este objetivo, la concertación de reuniones locales es otro de las razones de estas visitas. Destaca el bajo número de respuestas que registra la petición de favores personales en el caso de los diputados bolivianos.

Tabla 5 - Motivos más habituales (1er y $2^{\circ}$ motivo) de las visitas de electores al diputado en su oficina en el Congreso (en \%)

\begin{tabular}{|c|c|c|c|c|c|c|}
\hline & & MAS & PPB-CN & PDC & RN & PPD \\
\hline \multirow{2}{*}{$\begin{array}{c}\text { Informar sobre problema de la } \\
\text { circunscripción }\end{array}$} & $1^{\circ}$ & 92,9 & 46,7 & 66,7 & 42,9 & 66,7 \\
\hline & $2^{\circ}$ & & & & & \\
\hline \multirow{2}{*}{ Petición de favor personal } & $1^{\circ}$ & 3,6 & & 22,2 & 14,3 & 22,2 \\
\hline & $2 \circ$ & 5,0 & & 42,9 & & 25,0 \\
\hline \multirow{2}{*}{ Concertar algunareunión local } & $11^{\circ}$ & 3,6 & 20 & 11,1 & 14,3 & 11,1 \\
\hline & $2^{\circ}$ & 30 & 14,3 & 42,9 & 66,7 & 12,5 \\
\hline \multirow{2}{*}{$\begin{array}{l}\text { Informarse sobre estado de gestión o } \\
\text { trámite de ley }\end{array}$} & $1^{\circ}$ & & 26,7 & & & \\
\hline & $2^{\circ}$ & 65 & 85,7 & 14,3 & 33,3 & 62,5 \\
\hline \multirow{2}{*}{ Todas las anteriores } & $1^{\circ}$ & & 6,7 & & & \\
\hline & $2^{\circ}$ & & & & & \\
\hline \multirow{2}{*}{ No lo sé } & $1^{\circ}$ & & & & 28,6 & \\
\hline & $2^{\circ}$ & & & & & \\
\hline \multicolumn{2}{|l|}{$(\mathrm{N})$} & 28 & 15 & 9 & 7 & 10 \\
\hline
\end{tabular}

Fuente: Elaboración propia con datos del proyecto CSO2011-24344.

Con la información existente se puede decir que la actividad distrital es abundante y heterogénea. Sin embargo, con la evidencia disponible ninguno de los dos aspectos permite discriminar entre los cinco partidos analizados. 


\section{- Diputados leales al partido versus diputados servidores del distrito}

De las diferentes dimensiones de la actividad distrital susceptibles de análisis, la tensión entre el electorado y el partido constituye una de las posibles avenidas de estudio. Se presume que el diputado actúa condicionado por un triángulo de intereses (los del partido, los de su distrito y sus intereses personales) que debe intentar conciliar, priorizar u obviar en sus procesos de toma de decisiones ${ }^{4}$. No obstante, no se dispone de suficientes estudios que proporcionen una confirmación empírica de estas presunciones. Aunque es esperable que los partidos condicionen la actividad de los diputados en sus distritos, en el mapeo de la actividad distrital se encuentra que hay diputados que definen de forma autónoma las tareas que llevan a cabo en las circunscripciones por las que fueron elegidos. Sobre estas cuestiones nuestra expectativa inicial es que, además de la variedad de soportes e intensidad de la actividad distrital, existan diferencias en el grado de partidización de la actividad distrital entre partidos, así como en el interior de los mismos. Se espera que esta actividad distrital no sea homogénea en el interior de los partidos donde conviven diversos modos en que los diputados mantienen vínculos y llevan a cabo interacciones con su electorado.

Para contestar a estas cuestiones se introdujeron dos preguntas en la encuesta realizada a asesores de diputados. La primera pregunta solicita una valoración de con quién se relaciona más el diputado cuando está en su circunscripción: si con la sociedad civil o con su partido político. Se utiliza una escala de 1 (sociedad civil) a 10 (partido político). La segunda pregunta consiste en una valoración sobre quién influye más en los procesos de toma de decisiones del diputado: la sociedad civil o su partido. La escala es la misma que en el caso anterior.

Recurriendo a la tipología de diputados elaborada por Siavelis y Morgenstern (2008), se puede decir que cuanto más bajo sea el valor de un diputado en estas preguntas más se aproxima al perfil de "servidor de los votantes", mientras que cuanto más alto sea más se ajusta al perfil de "leal al partido". En la Tabla 6 se presentan los resultados entre los diputados de los principales partidos políticos de Bolivia y Chile. Como puede observarse el promedio de los diputados se ubica en la zona de "servidor de los votantes", pero sin llegar a situarse en posiciones extremas. De manera que los diputados, considerados en conjunto, cuando orientan su actividad de representación tienden a conceder mayor prioridad a las preferencias de los ciudadanos de su distrito que a las de sus partidos políticos. No obstante, si nos fijamos en la distribución de

\footnotetext{
${ }^{4}$ Por ejemplo, Eulau et al. (1959) y Thomassen (1994).
} 
los diputados por partidos se aprecian diferencias significativas. Así, los diputados bolivianos, especialmente los del MAS, son los que muestran los niveles más elevados de cercanía con el partido, en la situación opuesta se encuentran los diputados del PPD.

Tabla 6 - Autonomia con respecto al partido en la actividad distrital de los diputados

\begin{tabular}{c|c|c|c|c|c|c}
\hline & MAS & $\begin{array}{c}\text { PPB- } \\
\text { CN }\end{array}$ & DC & PPD & RN & Total \\
\hline Con quién se relaciona más el diputado & \multicolumn{5}{|l}{} \\
\hline Media (escala 1 soc. civil -10 partido político) & 4,4 & 3,5 & 2,8 & 2,2 & 3,3 & 3,6 \\
\hline Desviación típica & 2,2 & 2,2 & 0,8 & 1,3 & 0,7 & 1,9 \\
\hline $\begin{array}{l}\text { Quién le influye más en los procesos de } \\
\text { toma de decisiones }\end{array}$ & \multicolumn{7}{|l}{} \\
\hline $\begin{array}{c}\text { Media (escala 1 soc. civil -10 partido político) } \\
\text { Desviación típica }\end{array}$ & 4,9 & 4 & 2,9 & 2 & 3 & 3,8 \\
\hline (N) & 2,3 & 2,2 & 0,9 & 0,9 & 0,7 & 2,1 \\
\hline & 27 & 15 & 9 & 9 & 9 & 69 \\
\hline
\end{tabular}

Fuente: Elaboración propia con datos del proyecto CSO2011-24344.

El hecho de que los diputados del MAS sean los que presenten un nivel más elevado de partidización en su actividad distrital puede resultar, a primera vista, sorprendente. Como partido joven, con bajo nivel de institucionalización y creado como "instrumento político" al servicio de los movimientos sociales y organizaciones sindicales del mundo rural (ALTO y STEFANONI, 2009), cabría esperar, en principio, que tuviese unos niveles bajos de partidización y, sin embargo, ocurre justo lo contrario. El factor que puede explicar esta situación es la transformación del MAS en un partido de gobierno. La trayectoria del MAS desde una posición minoritaria hasta su mutación en un partido de gobierno ha comportado, entre otros aspectos, una fuerte concentración del poder del partido en manos de Evo Morales, el desarrollo de unas relaciones jerárquicas entre éste y los representantes en el Congreso (MAYORGA, 2008), y un cambio en las preferencias de los representantes. Aunque sigue siendo relevante la presencia de líderes campesinos en el Congreso, en éstos se ha constatado un proceso de profesionalización política y de burocratización, que les ha llevado a mantener un comportamiento más autónomo con respecto a sus organizaciones de base y más disciplinado con respecto al partido (ALTO, 2011) ${ }^{5}$.

${ }^{5}$ Uno de los elementos que propicia la cohesión en el MAS es la expectativa de conseguir puestos públicos: las llamadas "pegas" (ALTO y STEFANONI, 2009; ALTO, 2011). El gobierno de Evo 
Además de la variación interpartidos en el grado de autonomía de la actividad distrital, conviene considerar la variación en el interior de los partidos. De nuevo, los partidos bolivianos presentan una singularidad respecto a los chilenos: la disparidad del índice de partidización dentro del MAS y el PPB-CN es bastante superior (obsérvese cómo sus desviaciones típicas son mucho más elevadas). Hay una razón destacada que puede explicar estas diferencias: el tipo de circunscripciones. Como se ha resaltado en la literatura, las circunscripciones uninominales incentivan que la lealtad del representante se dirija más hacia los electores de su distrito que hacia su partido político (PERSSON, TABELLINI y TREBBI, 2003; COX, 2004; CARROLL y SHUGART, 2007). En Chile la influencia de la magnitud electoral no se evidencia, dado que todas las circunscripciones son del mismo tamaño (2). En cambio, en Bolivia hay circunscripciones uninominales y plurinonimales y dentro de estas últimas hay variaciones en cuanto a su magnitud. Es esperable, por tanto, que los diputados plurinominales del MAS y PPB-CN presenten unos niveles de partidización superiores a sus colegas uninominales.

Los datos sugieren que los dos partidos bolivianos están formados por diputados más leales al partido, de acuerdo con la tipología de Siavelis y Morgenstern (2008), pensada para candidatos y que aquí hemos trasladado para analizar los perfiles de los diputados. Por su parte, los partidos chilenos tienen un carácter más heterogéneo en su interior, de modo que no todos serían diputados que encajan en la caracterización de leales al partido en su actividad distrital.

\section{Determinantes de la autonomia partidista en la actividad distrital}

Como acabamos de ver, unos diputados son más autónomos que otros con respecto a sus partidos a la hora de desarrollar su actividad distrital. Pero ¿qué razones pueden explicar estas diferencias? En la literatura académica no encontramos unas explicaciones específicas para la actividad distrital, pero sí para la actividad de los diputados en otros ámbitos, que podemos tomar como referencia. En concreto, los estudios sobre representación han destacado tres grandes tipos de factores explicativos con potenciales efectos sobre la actividad distrital: las características de los representantes; los rasgos de los partidos políticos de los que son miembros; el entorno institucional y socioeconómico en el que operan los representantes.

Morales utiliza la distribución de pegas entre los miembros del MAS (incluidos los diputados) como incentivos selectivos para lograr la unidad en el partido y el apoyo al ejecutivo. 
Ante el reducido número de observaciones de nuestro estudio, el análisis estadístico se limita a examinar las correlaciones entre las dos variables que miden la tensión entre partido y diputado y un conjunto de variables explicativas. Se trata de un primer paso para comprobar en el futuro, de una manera más sólida, nuestra expectativa de que la actividad distrital constituye una estrategia del diputado que viene influida por variables relacionadas con sus propias características, con las de su partido político y con el entorno socioeconómico e institucional del distrito.

A continuación se detallan las variables explicativas que han sido seleccionadas y su justificación teórica. Se comenzará por las variables relativas a las características de los diputados.

\section{Caracteristicas de los diputados}

En la literatura se ha evidenciado el impacto de las variables relativas a los representantes sobre el funcionamiento de los partidos y sobre su comportamiento en la cámara. En el caso de la actividad distrital, la atención se centrará en cuatro variables referentes a los diputados.

La primera de las variables es la trayectoria política del diputado. Los trabajos de Carey (1998) y de Siavelis y Morgenstern (2008) proporcionan convincentes argumentos sobre los efectos en la concepción y actuaciones de los representantes reelectos frente a los recién elegidos. En esta misma línea, se argumenta que la imposibilidad de reelección incentiva que los legisladores dejen de actuar representando los intereses de los votantes y que orienten su lealtadhacia los líderes de los partidos y a otros actores capaces de garantizar sus carreras en el futuro (CARROLL y SHUGART, 2007). Estos efectos son trasladables a la actividad distrital. Sobre la base de estos planteamientos, se puede esperar que los diputados que lleven tiempo en el cargo tengan mayores incentivos para ser autónomos en relación con sus partidos.

La segunda y tercera variable se refieren a aspectos ideológicos. Por una parte, se incluye la sintonía ideológica del diputado con el partido político, ya que en la literatura sobre representación se ha destacado la importancia de atender a la proximidad existente entre las ideas y actitudes políticas del diputado y las de su partido. En general, se asume que la congruencia ideológica y programática favorece la cohesión interna del partido y la unidad en su comportamiento externo, por ejemplo en el parlamento (RUIZ, 2007). Resulta esperable que una mayor lejanía ideológica con el partido signifique una mayor autonomía con respecto del partido en la actividad distrital de los diputados. Por otra parte, se mide la relación entre el nivel de coincidencia de la ideología (en general, no sólo respecto al eje izquierda-derecha) 
del diputado con la de sus votantes y la autonomía partidista en el desarrollo de la actividad distrital. El sentido esperado de la relación es que a mayor coincidencia con la ideología del electorado haya más autonomía con respecto al partido.

Se ha incluido también una variable relativa al éxito electoral que cosecha el candidato en su distrito (votos que ha obtenido en relación con el número de votos emitidos en distrito). Es esperable que los candidatos exitosos tiendan a orientarse más hacia su electorado que hacia su partido, ya que su caudal de votos les lleva a sentirse legitimados para actuar de forma independiente con respecto al partido.

Finalmente, se comprobará la relación entre la autonomía en la actividad distrital y una quinta variable que tiene que ver con un instrumento de comunicación política de uso creciente: Internet.Algunos de los argumentos habituales sobre las potencialidades de Internet en el ámbito de la representación se pueden aplicar a la explicación de la autonomía partidista en la actividad distrital. Así, Internet abarata los costes de comunicación con los electores y contribuye a estrechar los vínculos entre éstos y sus representantes Además, de estos beneficios se pueden aprovechar todos los actores políticos, con independencia de sus diferentes recursos estratégicos (GIBSON et al., 2003; PADRÓ-SOLANET y CARDENAL, 2009). Por lo tanto, a través de Internet un diputado puede comunicarse de manera poco costosa, fluida y autónoma con los electores de su circunscripción. En este sentido, cabe esperar que el uso recurrente de Internet por parte del diputado favorezca una mayor autonomía con respecto al partido político en el desempeño de la actividad distrital.

\section{Rasgos de los partidos politicos de los diputados}

La literatura señala diversas variables relativas al partido político del diputado que afectan al modo en que se ejerce la representación y que es esperable que tengan impacto sobre el grado de autonomía de los diputados con respecto de los partidos en su actividad distrital. Aquí se centra la atención en cuatro variables.

La primera es el tamaño del partido político, medido a través del porcentaje de escaños en el parlamento. Alcántara (2004) en su medición del rendimiento electoral reivindicaba su impacto en la naturaleza de la representación. Es previsible que los diputados de partidos grandes tengan menor propensión a desarrollar la actividad distrital de representación de forma autónoma que los diputados de partidos pequeños; entre otras razones, porque los partidos grandes tienden a desarrollar estructuras organizativas complejas y a disponer de incentivos selectivos (por ejemplo, cargos públicos) que facilitan la unidad y la disciplina dentro del partido (MAINWARING, 1999). 
Relacionado con esta cuestión, se incluye como variable explicativa la condición de partido en el gobierno. Es esperable que los partidos en el gobierno tengan más recursos y los diputados se apoyen en ellos para llevar a cabo su actividad distrital. El caso comentado del MAS es muy ilustrativo de ello.

Las otras dos variables se refieren a la organización de los partidos políticos. En concreto, se toma en cuenta la pregunta sobre si el partido dispone de sede en la circunscripción del diputado. Cabe esperar que la existencia de una sede en el distrito favorezca una actividad política del diputado más alineada con las preferencias y orientaciones de su partido. Junto a ésta, se mide la relación con la democracia interna, en concreto con la percepción sobre el impacto de las cúpulas del partido en los procesos de toma de decisiones.

\section{Entorno institucional y socioeconómico de los distritos}

Existen variables relacionadas con el entorno institucional que tienen efectos sobre la representación y que pueden incidir en el tipo de actividad distrital. En este trabajo se comprueba el impacto de dos rasgos del entorno en el que se desenvuelven los diputados y que inciden en su actividad de representación.

La primera variable se refiere a un elemento del sistema electoral: la magnitud de la circunscripción electoral, quediversos autores han destacado por sus efectos políticos ${ }^{6}$. Es esperable que en las circunscripciones uninominales los diputados se sintiesen con mayor autonomía para desarrollar su tarea de representación, dado que tienden a percibir que el principal no es tanto su partido sino sus votantes (ellos son los que le eligen directamente y a quiénes debe rendir cuentas). La segunda variable es el nivel de desarrollo socioeconómico de cada circunscripción electoral. Un distrito con bajo nivel de desarrollo favorece el desarrollo de relaciones de intercambio político de tipo personal y clientelar (LUNA, 2007 y 2010; FOX, 1994). En este contexto, los diputados tienen mayor probabilidad de gestionar de manera más autónoma su agenda de representación en el distrito.

\footnotetext{
${ }^{6}$ Por ejemplo Cox (2004) y Lijphart (2000).
} 
Tabla 7 - Correlaciones entre el grado de autonomía partidista en la actividad distrital y variables relativas a los diputados, partidos y entorno de distritos

\begin{tabular}{c|c|c}
\hline & $\begin{array}{c}\text { Con quién se relaciona más } \\
\text { el diputado (a) }\end{array}$ & $\begin{array}{c}\text { Quién le influye más en los } \\
\text { procesos de toma decisiones } \\
\text { (a) }\end{array}$ \\
\hline $\begin{array}{c}\text { Con quién se relaciona más el } \\
\text { diputado }\end{array}$ & 1 & $0,69^{* *}$ \\
\hline $\begin{array}{c}\text { Quién le influye más en los procesos } \\
\text { de toma de decisiones. }\end{array}$ & $0,69^{* *}$ & 1 \\
\hline Primera legislatura diputado & $0,40^{* *}$ & 0,22 \\
\hline Lejanía ideológica con partido & 0,14 & 0,18 \\
\hline Coincidencia ideológica con votantes & $-0,05$ & $-0,01$ \\
\hline Éxitoelectoral & $0,47^{* *}$ & $0,34^{* *}$ \\
\hline Importancia de uso de Internet & $-0,23^{* *}$ & $-0,37^{* *}$ \\
\hline Tamańo partido (porcentajeescańos) & $0,45^{* *}$ & $0,35^{* *}$ \\
\hline Partido engobierno & $0,30^{*}$ & $0,29^{*}$ \\
\hline Partido con sede en distrito electoral & $-0,09$ & $-0,08$ \\
\hline Decisiones por cúpulas & $-0,39^{* *}$ & $-0,27^{*}$ \\
\hline Magnituddel distrito electoral & $0,37^{* *}$ & $0,31^{* *}$ \\
\hline Nivel de pobreza & $0,36^{* *}$ & 0,21 \\
\hline
\end{tabular}

(a) Los valores oscilan en una escala de 1 (sociedad civil) a 10 (partido político). $* \mathrm{y} * *$ indican niveles de significación del $95 \%$ y 99\%, respectivamente.

Fuente: Elaboración propia con datos del proyecto CSO2011-24344.

En la Tabla 7 se presentan los resultados del análisis de correlaciones. Como puede observarse, de las once variables independientes del modelo ocho son estadísticamente significativas con una o las dos variables que miden la tensión entre electorado y partido en la actividad distrital de los diputados.

Respecto al signo de los coeficientes, la mayor parte de las correlaciones significativas se ajustan a la argumentación planteada anteriormente. Así, la inexperiencia de un diputado (si es la primera legislatura del diputado), el uso limitado de Internet en su actividad distrital, la pertenencia a un partido fuerte (con grupo parlamentario grande) o en el gobierno y jerarquizado (en el partidotienden a mandarlas cúpulas) y la presencia de magnitudes de distrito grandes son factores que influyen en que el diputado actúe más como "leal al partido" que como "servidor al distrito" en su actividad distrital.

Junto a estos resultados, hay dos variables con una correlación que no era la esperada. El grado de éxito electoral del diputado y el nivel de pobreza existente en su distrito no presentan una correlación negativa, como se podía esperar, sino positiva. No obstante, esto no significa necesariamente que la justificación dada anteriormente sea incorrecta y deba ser revisada. Puede haber otras variables en juego cuyo efecto 
sería necesario controlar, pero que no resulta posible en un análisis de correlaciones. Así, por lo que se refiere al éxito electoral, puede ser que la variable experiencia electoral esté incidiendo en la correlación. Ciertamente, puede ocurrir que los diputados exitosos, que han sido reelegidos en más de una ocasión, interpreten su margen de victoria como resultado de su gestión más que de la etiqueta del partido al que pertenecen $y$, en consecuencia, tiendan a actuar con mayor autonomía respecto a su partido. En cuanto al nivel de pobreza, las variables referentes a la fortaleza del partido (por ejemplo, el tamaño del partido o si está o no en el gobierno) pueden estar afectando a la correlación. Así, los diputados elegidos en circunscripciones con altos niveles de pobreza y que forman de partidos fuertes pueden tener mayores incentivos a actuar como "servidores del partido", en la medida en que ello les facilita acceder a los diferentes recursos (económicos, humanos, informativos, etc.) que gestiona el partido.

Resulta llamativa la falta de relación de las variables referentes a la sintonía ideológica - ya sea con el partido o con el electorado - y la inclinación del diputado hacia ser servidor del distrito o servidor del partido. Ni la cercanía ideológica del diputado al partido influye en su inclinación hacia éste ni la sintonía ideológica entre electores y diputado le hace inclinarse hacia el electorado. De la misma forma, tampoco la existencia de sede en el distrito condiciona la relación del diputado con el partido y con el electorado.

\section{Conclusiones}

Este trabajo se ha ocupado de la actividad distrital de los diputados en períodos no electorales. El modo en que los diputados mantienen su presencia en las circunscripciones por las que fueron elegidos proporciona nueva evidencia empírica para profundizar en la comprensión del ejercicio de representación política.

De las posibles dimensiones de estudio de la actividad distrital la atención se ha centrado en el grado de autonomía de los diputados con respecto a sus partidos. La evidencia empírica utilizada se circunscribe a dos partidos bolivianos y tres partidos chilenos. Los datos ponen de manifiesto, por una parte, la diversidad de soportes a los que recurren los diputados en la actividad distrital. Por otra parte, los datos perfilan variaciones en el grado de dependencia de los diputados con respecto a sus partidos. Se aprecia que algunos diputados tienden a actuar como agentes del partido y otros como agentes de sus votantes. Los partidos bolivianos estarían formados por diputados que optan por una partidización de su actividad en los distritos, sobre todo en el caso del MAS. Mientras que los diputados chilenos elegirían estrategias mixtas que diversifican su actividad, vinculando unas al partido y otras de forma autónoma a 
éste. De los partidos chilenos, los diputados del PPD serían los menos dependientesdel partido en su actividad distrital.

Así mismo, en este trabajo se ha explorado la relación entre el grado de autonomía de los diputados con respecto a sus partidos y un conjunto de variables relacionadas con el diputado, el partido y el entorno. Se confirma la potencialidad de una comprensión multicausal del fenómeno. Existen aspectos relativos a los diputados que hacen que éstos se conviertan en servidores de los partidos en lugar de servidores de los distritos. Aquí se ha comprobado la relación entre la ausencia de trayectoria parlamentaria y un uso escaso de de nuevas tecnologías con la predisposición a ser un diputado leal al partido. En segundo lugar, se ha mostrado la relación entre pertenencia a un partido fuerte y jerarquizado y ser un diputado leal al partido. En tercer lugar, se ha evidenciado la influencia significativa de un elemento del sistema electoral: la magnitud de las circunscripciones. Así, las circunscripciones donde se elige a más candidatos incentivan a los diputados a operar como servidores del partido en el desarrollo de la actividad distrital. No obstante, todos estos resultados deben ser interpretados como unas primeras conclusiones, que deberán ser contrastadas, en el futuro, con estudios que contemplen más casos y que permitan aplicar análisis estadísticos más rigurosos.

Mikel Barreda é Professor da Universidade Aberta da Catalunha. E-mail:mbarreda@uoc.edu

Letícia M. Ruiz é Professora da Universidade Complutense de Madrid. E-mail: leticiamaria.ruiz@cps.ucm.es

\section{Referências}

ALCÁNTARA, Manuel. ¿Instituciones o máquinas ideológicas? Origen, programa y organización de los partidos políticos latinoamericanos. Barcelona: ICPS, 2004.

(Ed.) Politicians and Politics in Latin America.Boulder, CO., Lynne Rinner, 2007.

AMARAL, Samuel; STOKES, Susan. La democracia local en Argentina: Clientelismo, capital social, e innovación en Argentina. Buenos Aires: Universidad Tres de Febrero, 2005.

ALTO, Hervé do; STEFANONI, Pablo. El MAS: las ambivalencias de la democracia corporativa. Coloquio PNUD, Democracia interna en la elección de candidatos del Movimiento al Socialismo, La Paz, 2009. Disponible en: <http://www.mainumby.org.bo/wpcontent/uploads/2010/11/DoAltoStefanoni2010-MAS-PartidoHeterogeneo.pdf $>$. Aceso en: 20 mar. 2014. 
ALTO, Hervé do. Un partido campesino en el poder. Una mirada sociológica del MAS boliviano. Nueva Sociedad, n. 234, 2011. p. 95-111.

BRUSCO, Valeria; NAZARENO, Marcelo; STOKES, Susan. Vote-Buying in Argentina. Latin American Research Review, v. 39, n. 2, p. 66-88, 2004.

CAREY, John. Term limits and Legislative Representation. New York: Cambridge University Press, 1998.

Legislative voting and accountability. New York: Cambridge University Press, 2009.

CARROLL, Royce; SHUGART, Matthew S. In: MUNCK, Gerardo L. (Ed.). Neo-Madisonian Theory and Latin American Institutions. Regimes and Democracy in Latin America. Oxford: Oxford University Press, 2007. p. 51-101.

COX, Gary. La coordinación estratégica de los sistemas electorales del mundo: hacer que los votos cuenten. Barcelona: Gedisa, 2004.

EULAU, Heinz et al. The role of representative: some empirical observations on the theory of Edmund Burke. American Political Science Review, Cambridge, n. 53, p.742-756, sep. 1959.

FENNO, Richard F. Jr; STYLE, Home. House members in their districts. New York: Pearson, 2003. (Longman Lassics Series).

FOX, Jonathan. The difficult transition from Clientelism to Citizenship. Lessons from Mexico. World Politics, Princeton, v. 46, n. 2, p. 151-184, jan. 1994.

GIBSON, Rachel et al. Election Campaigning on the WWW in the USA and UK. A Comparative Analysis. Party Politics, v. 1, n. 9, p. 47-75, jan. 2003.

HAGOPIAN, Frances. Parties and voters in emerging democracies. In: BOIX, Carles, STOKES Susan (Eds.). The Oxford Handbook of Comparative Politics. Oxford: Oxford University Press, p. 582603, 2007.

HELMKE, Gretchen; LEVITSKY, Steven. (Eds.). Informal Institutions and Democracy: Lessons from Latin America. Baltimore: Johns Hopkins University Press, 2006.

HUNNEUS, Carlos. A highly institutionalized political party. In: MAINWARING, S.SCULLY, T. (Eds.). Cristian Democracy in Latin America. Electoral Competition and Regime Conflicts. Stanford: Stanford University Press, p. 121-161, 2003.

KITSCHELT, Herbert; HAWKINS, Kirk; LUNA, Juan Pablo; ROSAS, Guillermo; ZEICHMEISTER, Elizabeth. Latin American Party Systems. Cambridge: Cambridge University Press, 2010.

LIJPHART, Arend. Modelos de democracia: formas de gobierno y resultados en treinta y seis países. Barcelona: Ariel, 2000.

LUNA, Juan Pablo. Representación política en América Latina: el estado de la cuestión y una propuesta de agenda. Política y Gobierno, v. 14, n. 2, p. 391-435, 2007.

. Segmented Party-voter linkages in Latin America: the case of the UDI. Journal of Latin American Studies, v. 42, n. 2, p. 325-356, 2010.

LUNA, Juan Pablo; MARDONES, Rodrigo. Chile: Are the Parties Over? Journal of Democracy, v. 21, n. 3, p. 107-121, 2010.

MAINWARING, Scott. Party Systems in the Third Wave of Democratization. The Case of Brazil. Stanford: Stanford University Press. 1999.

MAINWARING, Scott; BEJARANO; Ana María. Eduardo Pizarro (Eds.). The Crisis of Democratic Representation in the Andes. Standford: Standford University Press, 2006. 
MAYORGA, René A. Populism in Bolivia: Can a Social Movement Govern without a Party? Artículo en Woodrow Wilson Center, 2008. Disponible en: <http://www.wilsoncenter.org/publication/bolivia-social-movements-populism-anddemocracy>.Aceso en: 20 mar. 2014.

MORGENSTERN, Scott; NACIF, Benito. (Eds.).Legislative politics in Latin America.Cambridge: Cambridge University Press, 2002.

NEGRETTO, Gabriel. Sistemas Electorales. In: BARREDA, Mikel (Coord.). Las instituciones politicas de las democracias latinoamericanas. Barcelona y La Paz: Huygens-Plural Editores, 2013. p. 95-136.

PADRÓ-SOLANET, Albert; CARDENAL, Ana S. Partidos y política en Internet: Un análisis de los websites de los partidos políticos catalanes. IDP. Revista de Internet, Derecho y Política, n. 6, p. 46-61, 2009. Disponible en: <http://idp.uoc.edu/ojs/index.php/idp/article/viewArticle/n6-padrocardenal>. Aceso en: 20 mar. 2014.

PELA. Proyecto de Élites Parlamentarias en América Latina. Universidad de Salamanca. http://americo.usal.es/oir/elites/>. Aceso en: 20 mar. 2014.

PERSSON, Torsten; TABELLINI, Guido; TREBBI, Francesco. Electoral Rules and Corruption. Journal of the European Economic Association, v. 1, n. 4, p. 958-989, 2003.

PLUMB, David. El Partidopor la Democracia. The Birth of Chile's Postmaterialist Catch-All Left. Party Politics, v. 4, n. 1, p. 93-106, 1998.

ROBERTS, Kenneth. Party-Society Linkages and Democratic Representation in Latin America. Canadian Journal of Latin American and Caribbean Studies, n. 53, p. 9-34, 2002.

RUIZ, Leticia M. Partidos y coherencia. Parlamentarios en América Latina. Madrid: Centro de Estudios Políticos y Constitucionales, 2007.

RUIZ, Leticia M. Partidos politicos y sistemas de partidos en las democracias latinoamericanas. In: BARREDA, Mikel. (Coord.). Las instituciones politicas de las democracias latinoamericanas, Barcelona y La Paz, Huygens-Plural Editores, p. 137-178, 2013.

SIAVELIS, Peter. MORGENSTERN, Scott (Eds.). Pathways to power: political recruitment and candidate selection in Latin America. University Park: Penn State University Press, 2008.

THOMASSEN, Jacques. Empirical Research into political representation: failing democracy or failing models. In: JENNINGS, K.; MANN, T. (Eds.). Elections at home and abroad. Essays in honor of Warren E. Miller. Ann Harbor: Michigan University Press, 1994.

TSE. Atlas electoral de Bolivia. Elecciones Generales 1979-2009. Asamblea Constituyente 2006. La Paz, OEP y PNUD: Tribunal Supremo Electoral, PNUD e IDEA Internacional 2010.

Texto recebido em 14 de dezembro 2013. Aprovado em 07 de março de 2014. 


\section{Anexo 1 - Ficha técnica trabajo de campo y partidos analizados}

Los cuestionarios se aplicaron a los asesores de los diputados mediante encuesta personal. Las encuestas contienen, en su mayor parte, preguntas cerradas y algunas preguntas abiertas ${ }^{7}$. La realización del trabajo de campo tuvo lugar durante los meses de febrero a abril de 2013.

\begin{tabular}{|c|c|c|c|c|c|c|c|}
\hline Siglas partido & $\begin{array}{c}\text { Nombredel } \\
\text { partido }\end{array}$ & $\begin{array}{c}\text { Ańocreacióndel } \\
\text { partido }\end{array}$ & País & $\begin{array}{c}\text { Legislatura } \\
\text { analizada }\end{array}$ & $\begin{array}{c}\text { Número } \\
\text { de } \\
\text { diputados }\end{array}$ & $\begin{array}{c}\text { Número } \\
\text { de } \\
\text { entrevistas } \\
\text { realizadas } \\
\text { entrevistas } \\
\text { realizadas } \\
\text { sobre el } \\
\text { total de } \\
\text { diputados }\end{array}$ \\
\hline MAS & $\begin{array}{c}\text { Movimiento al } \\
\text { Socialismo }\end{array}$ & 1995 & Bolivia & $2010-2015$ & 88 & 33 & 37,5 \\
\hline PPB - & $\begin{array}{c}\text { Plan Progreso } \\
\text { para Bolivia }- \\
\text { Convergencia }\end{array}$ & 2007 & Bolivia & $2010-2015$ & 37 & 13 & 35 \\
\hline PDC & $\begin{array}{c}\text { Partido } \\
\text { Demócrata }\end{array}$ & 1957 & Chile & $2009-2013$ & 19 & 9 & 47 \\
\hline PPD & $\begin{array}{c}\text { Partido por la } \\
\text { Democracia }\end{array}$ & 1987 & Chile & $2009-2013$ & 18 & & 9 \\
\hline RN & $\begin{array}{c}\text { Renovación } \\
\text { Nacional }\end{array}$ & 1987 & Chile & $2009-2013$ & 17 & & 7 \\
\hline
\end{tabular}

7 Se puede ver el modelo de cuestionario en: <http://edu.surveygizmo.com/s3/996569/Actividaddistrital-de-los-diputados-de-Chile-Per-y-Bolivia>. Aceso en: 30 nov. 2014. 


\section{Anexo 2 - Variables utilizadas en el análisis de correlaciones}

\begin{tabular}{|c|c|}
\hline Variable - Indicador & Fuente \\
\hline $\begin{array}{c}\text { Trayectoria política } \\
\text { Primera legislatura como diputado } \\
\text { Construcción e interpretación: Se asigna } 1 \text { a los casos en que es la primera legislatura del } \\
\text { diputado para el que trabaja y } 0 \text { a los casos en que no es así. }\end{array}$ & $\begin{array}{l}\text { Cuestionario sobre actividad } \\
\text { distrital a asesores (2013) }\end{array}$ \\
\hline $\begin{array}{c}\text { Distancia ideológica } \\
\text { Sintonía ideológica del diputado con el partido } \\
\begin{array}{l}\text { Construcción: Se calcula la diferencia entre la ubicación del diputado en la escala izquierda- } \\
\text { derecha y la ubicación del promedio de diputados de su partido. }\end{array} \\
\begin{array}{r}\text { Interpretación: Cuanto más elevados sean los valores de este índice hay menor sintonía } \\
\text { ideológica entre diputado y partido. }\end{array} \\
\text { Coincidencia ideológica con el electorado } \\
\text { Construcción: Se pide a los asesores que valoren el grado de coincidencia ideológica en } \\
\text { general (no sólo respecto al eje izquierda-derecha) del diputado en relación con sus votantes } \\
\text { Interpretación: Escala de valores de } 1 \text { (la ideología es prácticamente igual) a } 4 \text { (no coincide } \\
\text { en nada). }\end{array}$ & $\begin{array}{l}\text { Cuestionario sobre actividad } \\
\text { distrital a asesores (2013) }\end{array}$ \\
\hline 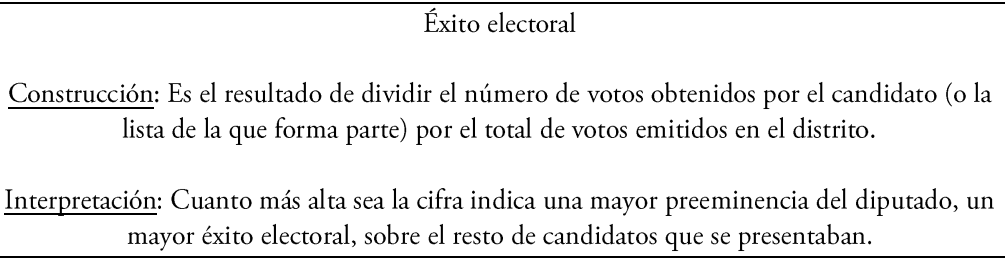 & $\begin{array}{l}\text { Cámara de Diputados de Chile y } \\
\text { Atlas Electoral de Bolivia (2010) }\end{array}$ \\
\hline $\begin{array}{l}\text { Importancia de uso de Internet } \\
\text { Construcción: La pregunta utilizada pide a los encuestados que valoren, la utilización de } \\
\text { Internet en el ejercicio de la actividad política del diputado en su circunscripción. } \\
\text { Interpretación: Escala de } 1 \text { (nada importante) a } 10 \text { (muy importante). }\end{array}$ & $\begin{array}{l}\text { Cuestionario sobre actividad } \\
\text { distrital a asesores (2013) }\end{array}$ \\
\hline $\begin{array}{l}\text { Tamaño del partido político } \\
\text { Construcción e interpretación: Porcentaje de escaños parlamentarios del partido al que } \\
\text { pertenece el diputado. }\end{array}$ & $\begin{array}{l}\text { Composición de las Cámaras de } \\
\text { Diputados de Chile (2010-2014) } \\
\text { y Bolivia (2010-2014) }\end{array}$ \\
\hline $\begin{array}{l}\text { Partido en el gobierno } \\
\text { Construcción e interpretación: Se asigna un } 0 \text { si el partido no está en el gobierno y un } 1 \text { si el } \\
\text { partido conforma el gobierno. }\end{array}$ & $\begin{array}{l}\text { Resultados elecciones } \\
\text { presidenciales }\end{array}$ \\
\hline $\begin{array}{l}\text { Partido con sede en el distrito } \\
\text { Construcción e interpretación: Se asigna un } 0 \text { si el partido no tiene sede en el distrito y un } 1 \\
\text { si el partido dispone de sede. }\end{array}$ & $\begin{array}{l}\text { Cuestionario sobre actividad } \\
\text { distrital a asesores (2013) }\end{array}$ \\
\hline
\end{tabular}




\section{Democracia interna \\ Poder de las cúpulas}

Construcción: La pregunta utilizada solicita a los diputados que señalen su grado de acuerdo con la afirmación de que en su partido las decisiones políticas las toma la cúpula y que las bases no pueden hacer oir su opinión.

Interpretación: Se utiliza una escala donde 1 es muy en desacuerdo y 5 muy de acuerdo

Magnitud del distrito

Construcción e interpretación: Número de diputados que se eligen en el distrito por el que fue elegido el diputado.

Grado de desarrollo socioeconómico

Nivel de pobreza Construcción e interpretación: Porcentaje de población bajo el umbral de la pobreza.

Cámara de Diputados de Chile y Atlas Electoral de Bolivia (2010)

SINIM de Chile y Fundación Milenio de Bolivia (a partir de

PELA de Bolivia (estudio 81) y PELA de Chile (estudio 77)

datos de INE) 
